\title{
The Innovation of the Public Sector through Artificial Intelligence - Analysis
}

\author{
Cosmina Crăciunescu Timofte PhD \\ Babeș-Bolyai University \\ 103 Axholme Avenue, Edgware, HA8 5BD \\ London, England, The United Kingdom
}

\begin{abstract}
The innovation of the public sector is an ongoing process, as the public arena is constrained to comply with the new rules and regulations that come into place due to the introduction of the most advanced technologies. The OECD (Organization for Economic Cooperation and Development) adopted the Declaration on Public Sector on May 22nd, 2019. (Santos, 2019) The purpose of the present article is the creation of a qualitative analysis on the topic of public sector innovation. The analysis should provide new ways of coping with the problems of the society, as the governments are faced with more complex challenges, in comparison to the past years. In addition, it is important to take into account the fact that the governments are compelled to think differently and find new solutions and design structures for the administrative systems. In this respect, innovation becomes a matter of necessity, rather than a viable option.
\end{abstract}

Keywords: innovation, public sector, artificial intelligence.

\section{Overview}

Since the introduction of artificial intelligence in most of the employment areas, the newest technologies brought about a revolution within the public sector. Starting with the 1950s, when artificial intelligence was created, the world entered a new phase, where all the processes started to go through automation. Eventually, automation became one of the most pressing needs of the modern society, due to the fact that the flow of information is very fast, and the pace of living escalated quickly during the past years. Along with the insertion of artificial intelligence, the functionalities of the society and the companies changed, calling for innovation in multiple sectors. In this respect, the processes of automation were introduced nearly everywhere, starting in the most advanced areas of the world, such as Western Europe, The United States and the United Arab Emirates.

The public sector is going through a phase of renewal for the past years, as more technologies were implemented, eventually answering to the needs of the customers. It is important to take into consideration the fact that the public sector is still flawed, and that there are still plenty if issues that could be addressed through the introduction of artificial intelligence. Some of the examples that could be brought to light are the bureaucracy and the waiting lines for processing different requests and documents.

\section{Methodology}

The method used for the present research article is qualitative. This method was chosen for the purpose of the creation of a qualitative analysis in the context of innovating the public sector through the introduction of artificial intelligence.

The qualitative method focuses on obtaining data through the use of open ended and conversational communication. It is about answering some of the most pressing questions of the society, involving systematic observation and analysis. For the present article, qualitative data analysis will be employed. The qualitative data analysis refers to the extraction of meaning from phenomena that it is placed under study. An assessment of the innovation of the public through the introduction of artificial intelligence will be presented over the course of the article. The types of social assessment are also connected to the application of qualitative methodology, being combined in the article as a form of practical analysis. The research goal: Assessing the innovation of the public sector in the context of introducing artificial intelligence.

\section{Theoretical Aspects}

Artificial Intelligence refers to automation that goes beyond the physical, the automation of the tasks that previously took a human brain to be completed, such as conversation, data analysis and other activities. (Miller, 2017).

One of the earliest areas of government application is in customer service chatbots. (Miller, 2017) The state and the local governments started making information available on the websites in the past decades, as they found they could use portals as means of answering the queries of the citizens. 
This action eventually brought good results in the sense that the government offices did not have as many calls to answer during the day, as they could devote more time and resources in other functions. (Miller, 2017)

The use of Artificial Intelligence in this case proved highly efficient, due to the fact that the majority of the customers called for the purpose of changing their passwords. Moreover, in the circumstance when the situation involved having a customer present for a problem solving matter, AI could streamline the process by getting information from a caller and wait for somebody to take their call. (Miller, 2017)

More and more governments started to adopt the introduction of artificial intelligence, in Los Angeles and Mississippi, people can use a city built chat bot that answers business-related questions for citizens. Moreover, the Amazon Alexa artificial intelligence robots can be used in the state of Mississippi in order to plug into the government information about taxes and registration of vehicles. (Miller, 2017)

The most important problem that surfaces in this situation is connected to the bureaucracy, representing a real problem in the public system. But there is a great opportunity for change in the bureaucratic world along with the introduction of artificial intelligence. The uses of the newest technologies could be multiple when it comes to their application and implementation in the public systems. Among these could be named: identifying the spread of diseases with ease, generating dynamic data dashboards to answer the questions of the citizens, creating a system of "listening" to social media in order to gather the public feedback on government-related topics, checking into various systems in order to find the problem coming from the servers. Other uses could be connected to the traffic systems, as the enabling of selfdriving shuttles could bring passengers to or from transit stations. (Miller, 2017)

With regard to the innovation of the public sector through artificial intelligence, the UN report of 2019 covers various AI projects that are piloted, implemented or planned by the governments across three applications, namely, citizen engagement, improvement of the operational efficiency, AI strategies for policy and legislation. The AI applications in government are nascent at the present time, as the governments need to overcome several hurdles in order to successfully adopt the new technologies. (Bharadwaj, 2019)

With regard to the AI applications, the government leaders will need to invest in the upgrade of their legacy within the IT systems. Eventually, the failure of doing so, may result in various compatibility issues at the point where the software need to be integrated. On the other hand, the leaders of the governments will have to make sure their departments have the necessary computing resources for an AI project. This implies the introduction of the cloud computing solutions, but the fact that more expensive technology needs to be used is another aspect worth considering. (Bharadwaj, 2019)

As for the present time, there is a number of projects which are developing, involving the use of artificial intelligence, with the aim of innovating the public sector. These projects are applied in the United States and are related to the following issues:

\section{Citizen Engagement}

For the Social Services pertaining to the New York City area, the Department currently has a staff of 18,000, offering public assistance and employment services to more than three million citizens. Due to the fact that the staff is unable to handle such a large number of papers (about 70,000 per month), the Department for Social Services needed to digitalize and automate the process, in an attempt to offer a self-service through an online portal, which is named ACCESS HRA (or AHRA). (Bharadwaj, 2019) The AHRA portal had as a goal the improvement of the online experience for website visitors and at the same time, reducing the number of people entering the centers of brick and mortar. The portal allows users to ask questions concerning their disability benefits, along with income support, food assistance and health insurance. (Bharadwaj, 2019)

\section{Citizen and Immigration Services}

The US Citizenship and Immigration Services (USCIS) announced back in 2015 the launch of a chatbot named Emma. The robot is able to answer questions related to immigration and take visitors on the right page of the official website of the Immigration Services. (Bharadwaj, 2019) In order to be able to function properly, the robots use natural language processing along with natural language generation. The algorithm was set up on thousands of questions related to various common immigration questions, along with requests for services, such as visa applications. (Bharadwaj, 2019)

\section{Automated Traffic Control - Improving the Operational Efficiency with Artificial Intelligence}

The city of Pittsburg made a collaboration agreement with the Rapid Flow Technologies in order to develop SUTRAC (or the Scalable Urban Traffic Control), which is known as an automated traffic oprimization and control software. The city traffic control departments are able to use the SUTRAC in order to manage traffic flows through several intersections, and make use of Artificial Intelligence in order to optimize the traffic systems. (Bharadwaj, 2019). 


\section{Predicting Fire Risk in Buildings}

The researchers from Georgia Institute of Technology, who are specialized on AI, came up with the idea of building and developing a predictive analytics software, aiming at identifying the buildings that have a higher likelihood of fire incidents. (Bharawaj, 2019) On the other hand, a group of researchers developed a software named "Firebird", which is using historical data for 58 variables made available to them by the AFRD. This is including the data related to the location of the properties, fire incidence, along with the size of the buildings, the structures and the year built for the time period between 2010 and 2014. The data was transferred to an AI predictive analytics software and the algorithms were programmed in such a way as to forecast risk scores for fires in 5,000 buildings. The software accurately predicted $73 \%$ of the fire incidents that took place in the building. (Bharawaj, 2019)

The current uses along with the potential of Artificial Intelligence in connection to the issue of innovating the public sector are referring to aspects such as:

- Object recognition, or computer vision, which allows the processing of information from digital images or videos. The computer vision AI is able to recognize unique features in order to identify objects, actions or unique characteristics.

- Pattern recognition, which allows for more accurate and comprehensive predictions by the use of very large amounts of data.

- Detection of anomalies, in which the use of AI is highlighting cases that stand out from other data sets that are larger in nature

- Natural Language Processing (NLP), which allows understanding of audio and text options for interactive communication.

- Profiling and grouping of citizens that have similar needs or interests in order to enable the provision of customized public services. (AI Watch, European Commission, 2019)

The use of Artificial Intelligence is able to provide many benefits to society, at the same time leading to unintended consequences in the instance when it is not handled properly.

\section{Qualitative Analysis of the Innovation of the Public Sector through Artificial Intelligence}

The analysis employed for the present article is qualitative, more specifically, it is a hermeneutic type of analysis. The hermeneutic analysis is known to be the one in which the researcher is making an effort to interpret the subjective meaning of a given text within a socio-historic context.

In the circumstance when the innovation of the public sector is directly connected to the ways the government makes decisions, it is important to bring to light the relationship between the two bodies. In this respect, the public sector is deeply affected by the introduction of artificial intelligence in the lives of the people and the functions of the institutions. Due to the fact that the machine learning strategies are rapidly evolving, the administrative tasks will eventually become automated. The automation of the public sector will have as an effect the boosting of the efficiency of institutions, at the same time allowing the public workers to focus on more important problems. As for the 20192020 period of time, one has to take into account the fact that it is also the time set up by the European Union to improve the quality of de delivered services with the help of the most advanced technologies. As the demand for more efficient technologies increases, the need for having a more homogenous public sector, relying on efficient technology and artificial intelligence is also met, for the purpose of delivering the best results for the citizens.

At the level of bureaucracy, it is important to stress on the fact that the introduction of artificial intelligence will have the capacity of easing the processes that involve a large quantity of paperwork. Bureaucracy represents one of the most pressing issues of the administrative systems, becoming a burden for almost every public entity, as well as for the citizens. Artificial intelligence has the potential to represent the breakthrough that is needed at the level of society, in relation to bureaucracy. The paperwork could be manipulated via intelligent machines, in a more accurate and timely manner, eliminating the human error and contributing to the efficiency of the bureaucratic and legal processes, which are connected to the documents and various public issues that need efficient resolution. If it is to take into consideration the issues centered around the citizen services, the introduction of artificial intelligence made for the purpose of serving the community, brings about a great change. The innovation that comes along with the implementation of the new technology is also considered a significant catalyst for modernization. The use of artificial intelligence in the services delivered for the citizens proved its potential of speeding the lengthy processes for the handling of the documents and paperwork, solving citizen inquiries in a timely manner. Moreover, the citizens are provided with a palette of options that can be customized to fit their needs. AI can interfere with every aspect of public administration and citizen services, ranging from automating processes of registration to identifying fraud and criminal activity. 
When it comes to the issue of traffic control, the introduction of artificial intelligence is also responsible for bringing about significant changes. The Smart Mobility is known to connect road users to the information systems. With the exception of anonymous road side traffic information, the data extracted from the individual vehicles will help road users to make better decisions. The level of autonomy in the present context, with the involvement of artificial intelligence in autonomous vehicles is rising, due to the progress that is made in the manufacturing of auto pilots. (Dynniq, 2019)

There are multiple benefits following the introduction of smart mobility in the society, due to the fact that it opens up the possibilities for a safer traffic system, along with facilitating the accessibility and livability of cities. The priority allocated to the public transportation systems will eventually lead to predictable time schedules. The trucks will be guided through or around the city in the most efficient ways for the purpose of conserving fuel and emissions. (Dynniq, 2019)

\section{Conclusions}

The innovation of the public sector is more accessible than ever with the introduction of artificial intelligence. It came as a great necessity for the public, especially when it comes to the delivery of public services and the well-being of the citizens. In addition, innovation meets the current needs of the people, as the technologies evolve to higher standards. It is important to understand the fact that the innovation of the public sector is directly influenced by the decisions that are made at the level of governance. The automation of the processes will most certainly bring about a significant boost to the overall economy, increasing the efficiency for the delivery of services.

Among the issues that can be tackled in relation to the innovation of the public sector are those related to the engagement of the citizens in the public life, at the same time learning to work with artificial intelligence. The article showed that the use of artificial intelligence might improve the interaction with the customers and citizens, through the introduction of virtual assistants that would be able to answer some of the most common queries of the people.

Lastly, the introduction of automated traffic control systems will prove to be of significant help for the society, contributing to less accidents and the creation of a safer traffic environment for both the people and the vehicles.

In conclusion, the innovation of the public sector is a continuous and necessary process, due to the fact that it has to be permanently connected with the waves of advancements that are taking place in the world. Technology and artificial intelligence both play significant roles in the area, being essential catalysts for change.

\section{Bibliography}

Bharadwaj, R., 'AI in Government - Current AI Projects in the Public Sector', Emerj, https://emerj.com/ai-sectoroverviews/ai-government-current-ai-projects-public-sector/ (Accessed August 26, 2019)

European Commission, 'AI in the Public Sector', https://ec.europa.eu/knowledge4policy/ai-watch/topic/ai-publicsector_en(Accessed August 26, 2019)

Miller, B., 'Automation Beyond the Physical: AI in the Public Sector', Government Technology, https://www.govtech.com/civic/GT-September-Automation-Beyond-the-Physical-AI-in-the-PublicSector.html(Accessed August 25, 2019)

Observatory of Public Sector Innovation, https://oecd-opsi.org/projects/innovationdeclaration-2/ (Accessed Aug 22, 2019)

Santos, R., 'It's official: The OECD Declaration on Public Sector was adopted today!' https://oecd-opsi.org/its-officialthe-oecd-declaration-on-public-sector-adopted-today/ (Accessed Aug 21, 2019)

Smart Mobility, https://dynniq.com/innovation/smart-mobility/?gclid=CjwKCAjwtajrBRBVEiwA8w2Q8Ol-xJ_ O0eV05 EE5nCANtkqblt95hurjq_p5w4TZ9SSDsG1YkRtDnBoCOdsQAvD_BwE (Accessed Aug 30, 2019) 